\title{
THE SEARCH FOR EFFECTIVE ALGORITHMS FOR RECOVERY FROM LOSS OF SEPARATION
}

\author{
Ricky Butler, George Hagen, Jeffrey Maddalon, César Muñoz, Anthony Narkawicz \\ NASA, Hampton, Virginia
}

\begin{abstract}
Our previous work presented an approach for developing high confidence algorithms for recovering aircraft from loss of separation situations. The correctness theorems for the algorithms relied on several key assumptions, namely that state data for all local aircraft is perfectly known, that resolution maneuvers can be achieved instantaneously, and that all aircraft compute resolutions using exactly the same data. Experiments showed that these assumptions were adequate in cases where the aircraft are far away from losing separation, but are insufficient when the aircraft have already lost separation. This paper describes the results of this experimentation and proposes a new criteria specification for loss of separation recovery that preserves the formal safety properties of the previous criteria while overcoming some key limitations. Candidate algorithms that satisfy the new criteria are presented.
\end{abstract}

\section{Introduction}

Distributed self-separation is an air traffic management concept where individual aircraft make independent decisions about how to resolve conflicts they encounter [1]. Due to concerns about pilot workload, efficiency, and situational awareness, these decisions are often accomplished through sophisticated automation. In our previous work [2, 3], algorithms that recover from loss of separation were developed along with a verification approach to obtain high confidence in the safety of these algorithms. Furthermore, we presented an approach that enables the aircraft to use different loss of separation recovery algorithms while still guaranteeing a safe recovery, even when aircraft simultaneously maneuver in response to a loss of separation situation. This safety property is called implicit coordination. The approach is based on the concept of criteria that each algorithm must satisfy. A formal proof was developed that shows that meeting the criteria is sufficient to guarantee implicit coordination. The important consequence is that any algorithm that satisfies the criteria will safely operate with any other algorithm that also satisfies the criteria.

The original criteria specification for loss of separation (LoS) recovery was based on the concept of divergence. Two aircraft are in divergent trajectories if the distance between the aircraft monotonically increases. Loss of separation algorithms that satisfy the divergence criteria are guaranteed to yield divergent trajectories, when one or both aircraft maneuver according to the algorithms. Through experimentation, it was discovered that the correctness property of divergence did not match real-world expectations and that the loss of separation algorithms based on the concept of divergence depended on some overly optimistic assumptions. In this paper, we present the results of the deficiencies discovered in the earlier approach and a revised criteria that solves most of the problems encountered.

We have developed a state-based, multi-aircraft conflict detection and resolution computer program called Chorus based upon our criteria. This program seeks to compute resolution maneuvers that avoid conflicts with all aircraft within a specified lookahead time. The program gives special attention to the most urgent conflict, that is, the conflict that has the smallest time to loss of separation or, in the case of LoS, the aircraft that is nearest at the time of closest approach. An implicitly coordinated resolution maneuver with respect to the most urgent aircraft is returned. The program also seeks to make this resolution free of secondary conflicts, that is, the resolution maneuver is free of traffic conflicts within the specified lookahead time.

\section{Notation}

The criteria approach assumes that both the position and velocity vectors of the aircraft are known. Vector variables are written in boldface, e.g., $\mathbf{v}$, and their components are referenced by sub- 
indices, e.g., $v_{x}, v_{y}$, and $v_{z}$. Position and velocity vectors for the ownship are denoted $\mathbf{s}_{\mathbf{0}}$ and $\mathbf{v}_{\mathbf{0}}$, respectively. Traffic vectors are denoted $\mathbf{s}_{\mathbf{i}}$ and $\mathbf{v}_{\mathbf{i}}$, and resolution velocity vectors are denoted by primed variables, e.g., $\mathbf{v}_{\mathbf{0}}^{\prime}$ and $\mathbf{v}_{\mathbf{i}}^{\mathbf{\prime}}$. We often use a relative coordinate system where the traffic aircraft is located at the origin of the system and is motionless. The relative position and velocity vectors of the ownship, with respect to the intruder, are denoted $\mathbf{s}$ and $\mathbf{v}$, respectively, where $\mathbf{s}=\mathbf{s}_{\mathbf{0}}-\mathbf{s}_{\mathbf{i}}$ and $\mathbf{v}=\mathbf{v}_{\mathbf{0}}-\mathbf{v}_{\mathbf{i}}$.

\section{The Criteria Approach}

A loss of separation occurs when two aircraft are both horizontally and vertically closer than prespecified limits. Aircraft in a loss of separation must maneuver to recover separation. Our approach to guaranteeing recovery of such maneuvers is based upon the concept of a criteria specification layer shown Figure 1.
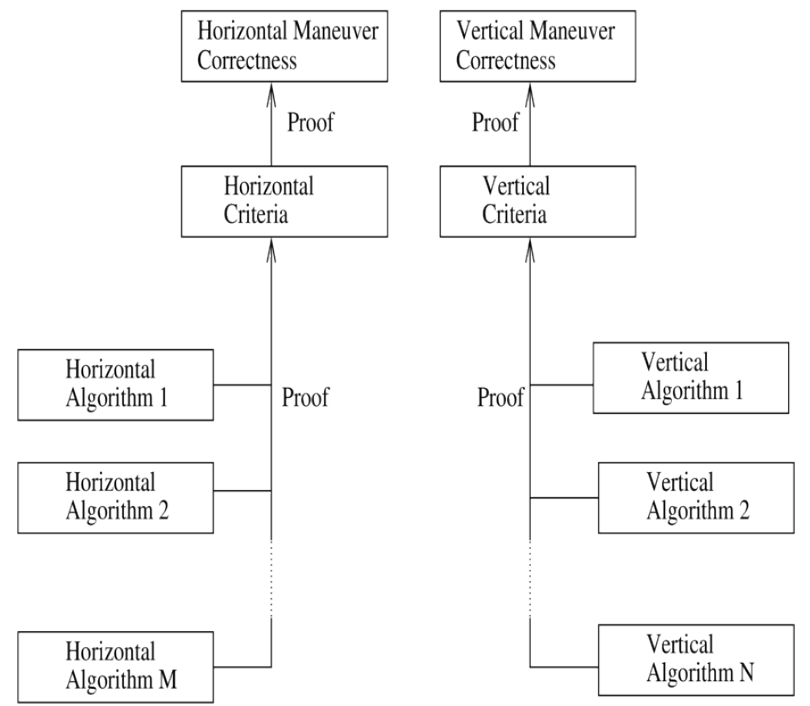

Figure 1. Criteria-based algorithm verification

The correctness properties associated with the horizontal and vertical maneuvers insure both independent and coordinated correctness. In other words, safe operation is guaranteed if only one aircraft maneuvers or if both aircraft maneuver. The intermediate specification layer is called criteria. We have separate concepts for horizontal and vertical correctness, and therefore separate criteria for the horizontal and vertical dimensions as well. There is a formal proof that the horizontal criteria satisfy the horizontal correctness property and a formal proof that the vertical criteria satisfies the vertical correctness property. Many different algorithms can then be shown to satisfy the criteria and thereby inherit the associated correctness properties. One interesting consequence is that all possible combinations of the algorithms that meet the criteria will have coordinated solutions: each aircraft can execute its own, possibly proprietary, algorithm as long as it satisfies the criteria.

The original concept of correctness for loss of separation recovery was centered on the idea of divergence and timeliness. The correctness theorems depend upon the following assumptions: (1) that aircraft state data is perfectly known, (2) the solution vectors can be achieved instantaneously, and (3) all aircraft compute their solutions using exactly the same data. In many real-world situations these assumptions are invalid. A key validation task is to assess the operational consequence when these assumptions are violated. We have found that in a conflict situation, i.e., when aircraft are predicted to lose separation within a given lookahead time, violations of these assumptions do not significantly change the resolution: because there is often time to correct initial errors. However, in a loss of separation situation these assumptions are too strong and there may not be enough time to make corrections. This paper explores how we account for discrepancies.

The original criteria for both horizontal and vertical recovery required divergence, where horizontal divergence is defined as follows: xy_divergent?(s,v) $\equiv \forall t: t>0 \Longrightarrow\|\mathbf{s}\|<\|\mathbf{s}+t \mathbf{v}\|$.

This property means that for all time in the future, the relative horizontal distance between the aircraft is greater than it was initially. In a similar manner, the vertical divergence is defined as follows: z_divergent?(s,v) $\equiv \forall t: t>0 \Longrightarrow\left|s_{z}\right|<\left|s_{z}+v_{z} t\right|$.

\section{Evidence Of Insufficiency}

In the fall of 2011, we began to experimentally validate our loss of separation algorithms implemented in the Chorus program using simple kinematic models for the aircraft trajectories. Although there were many situations where our algorithms performed well, there were other situations where they performed poorly. These 
situations primarily fell into two categories: inappropriateness of some of our assumptions and of the divergence property for generating achievable resolutions, and discontinuities observed in suggested resolutions as they evolved over time. More specifically, the following deficiencies were discovered:

1. Maneuvers could be overly restricted by the divergence property, occasionally resulting in unreasonably severe or even non-existent resolutions. For example, there are cases where a turn much smaller than the one calculated is sufficient to recover from loss of separation.

2. How one achieves a maneuver is more important to overall safety than the maneuver goal. The divergence criteria did not specify a turn direction and in some cases, the minimal turn direction is not the best.

3. Occasionally divergence would occur within a few seconds without maneuvering and yet our approach produced poor results because it only sought solutions that immediately achieved divergence.

4. The switch from conflict avoidance to loss of separation recovery would sometimes result in radical changes in the calculated resolutions.

5. In some cases, the resolutions would switch direction back and forth as time progressed. This lack of continuity led us to add requirements about sequences of resolutions over time that we had originally not foreseen.

The rest of this section shows scenarios that illustrate the deficiencies enumerated above.

\section{Overly Restricted Maneuvers}

As we explored the criteria concept with hundreds of test cases, we began to notice that there were situations where the maneuvers were extreme when using our original criteria for recovery from loss of separation.

Figure 2 illustrates this situation. The green area indicates the allowed region of track resolutions, and the red area indicates the disallowed region. The longer black and magenta vectors show the current velocity vectors of the ownship and intruder, respectively. For both aircraft the allowed maneuvers are quite severe.
If the original track of the traffic aircraft is changed to 210 degrees, then the consequence is shown in Figure 3 where the blue region indicates acceptable vectors. The situation has changed significantly. As indicated by the figure, one aircraft no longer has any track solutions available. Admittedly, this is a fairly extreme example in that the loss of separation is significant - the aircraft are only $1.97 \mathrm{~nm}$ apart. However, it does illustrate the fact that divergence is probably too strong of a notion of correctness.

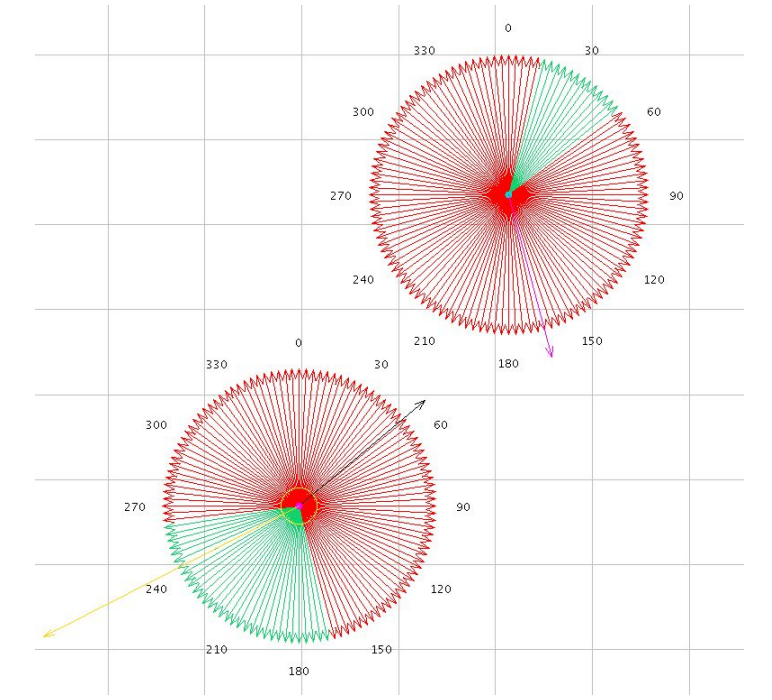

Figure 2. Criteria for LoS: Extreme Maneuver

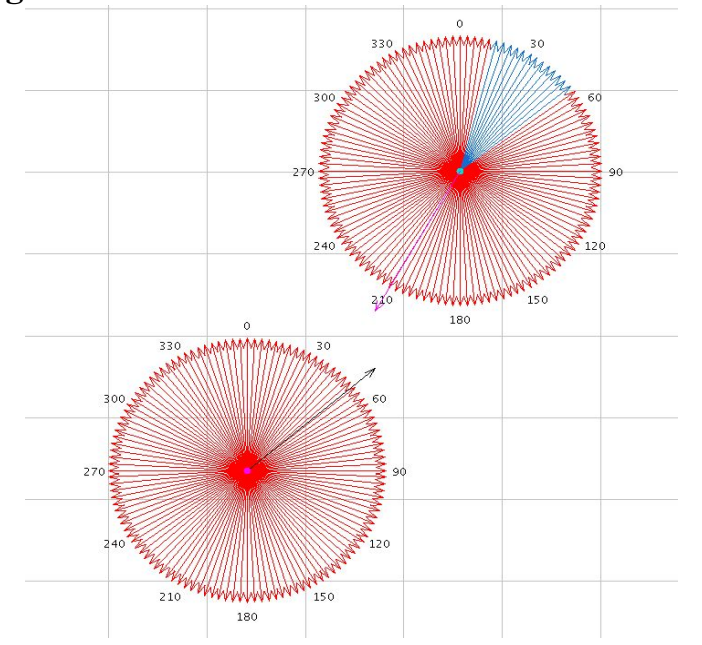

Figure 3. Horizontal Divergence Criteria

We have also discovered situations where no horizontal maneuvers were obtained even where the current separation is over four nautical miles. This can occur when there is a large difference between 
the ground speeds of the aircraft. In this case, the slower aircraft may have no maneuvers available that avoid conflict. This scenario is illustrated in Figure 4.

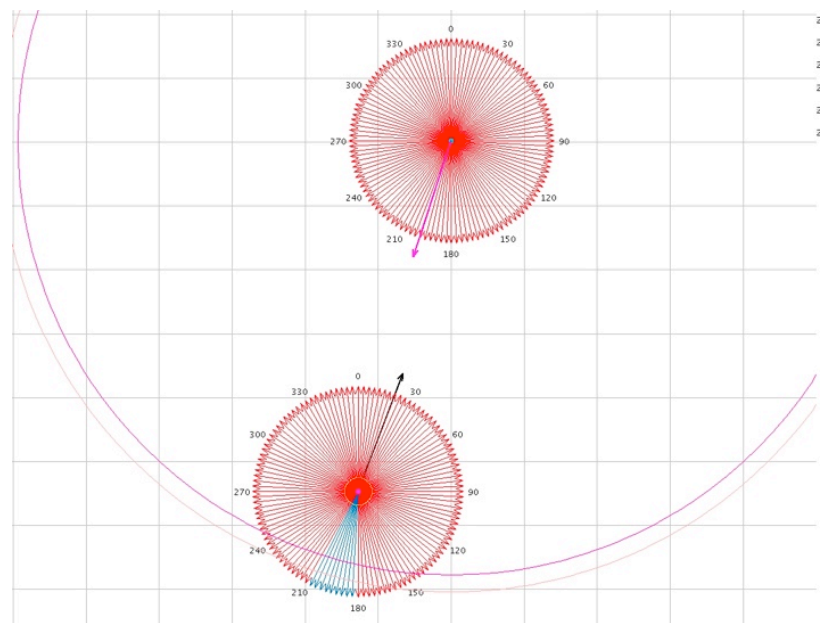

Figure 4. No Horizontal Resolutions

\section{Impact of Ignoring Maneuver Direction}

The original criteria only presented a set of resolutions, not the direction to achieve a resolution. If the maneuver could be achieved instantaneously, as our original proofs assumed, then the direction of the maneuver would be irrelevant.

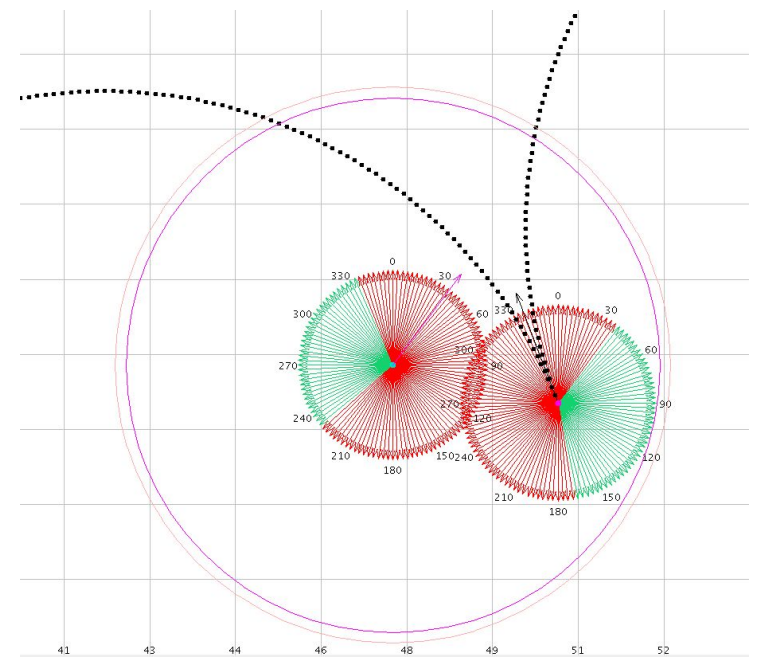

Figure 5. Impact of Turn Maneuver

The dotted line in Figure 5 shows the path of the ownship for both left and right turns. The aircraft can turn either left or right to reach the allowed green region. In this scenario, it is clear that the left maneuver requires a much greater time to achieve the goal track. In fact, recovery from the loss of separation will be achieved long before the goal track is reached. It is also not clear which turn direction is best. In fact, if the traffic aircraft does not maneuver, then the distance at the closest point of approach is closer if the aircraft turns right, i.e., left turn: $0.44 \mathrm{~nm}$ vs. right turn: $0.19 \mathrm{~nm}$. If neither aircraft maneuvers, the aircraft will be $0.18 \mathrm{~nm}$ at the closest point, which is only slightly worse than the right turn case. This is unfortunate because it is usually preferable to choose the smaller maneuver. ${ }^{1}$ But, what if the traffic aircraft also maneuvers? It is not the final track that matters, but rather the direction of the turn that determines how close the aircraft become. This suggests that the notion of correctness for loss of separation should be concerned primarily about the direction of the turns and not the goal tracks. Figure 6 shows an example where the path to the final divergent solution tracks brings the aircraft within $0.01 \mathrm{~nm}$ of each other.

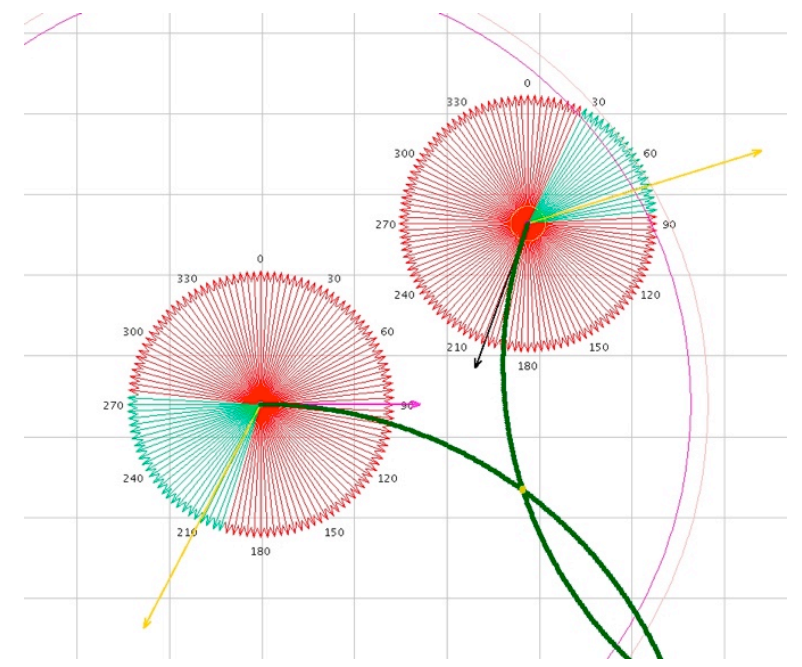

Figure 6. Close Encounter

The gold dot on the trajectories shows the future point of closest approach. The turns were calculated using the following simple kinematic model that defines a turn with radius $R$ :

$$
R=\frac{v^{2}}{g \tan \varphi}
$$

where $v$ is the ground speed of the aircraft, $g$ is the gravitational acceleration, and $\varphi$ is the bank angle of the aircraft.

A similar problem occurs for vertical maneuvers. The vertical path of the aircraft under a constant acceleration is shown in Figure 7. In this figure the aircraft is accelerating from a negative

\footnotetext{
${ }^{1}$ This is what our ACCoRD CRSS program does.
} 
vertical speed to a positive vertical speed. In this case, the impact is minimal because the aircraft have a large horizontal separation.

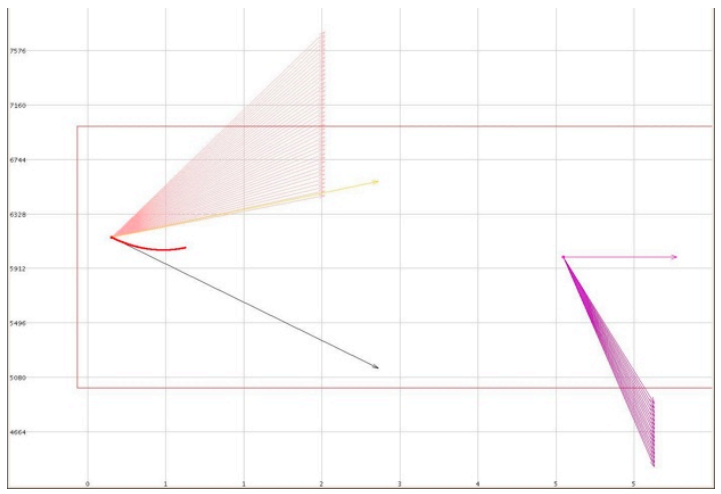

Figure 7. Vertical Path Of The Aircraft Under Constant Acceleration

The case where the horizontal separation is small and an aircraft is descending on top of another aircraft is especially critical. This case is illustrated in Figure 8. In this case it is unclear whether the safe maneuver is to go up or to go down. Answering this question relies on a fairly accurate model of the performance of both aircraft. The necessity of aircraft performance knowledge is illustrated in Figure 9 by using the same scenario, but with the acceleration cut in half.

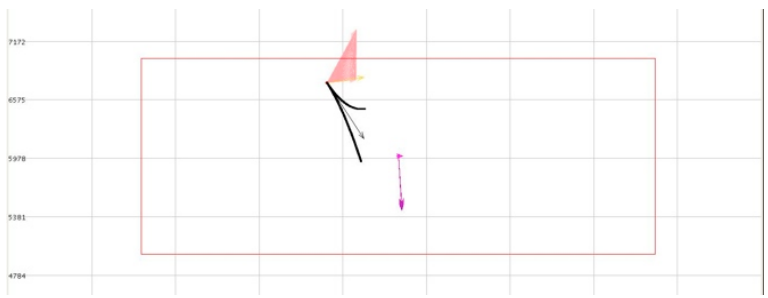

Figure 8. Impact of Acceleration On Vertical Maneuvers

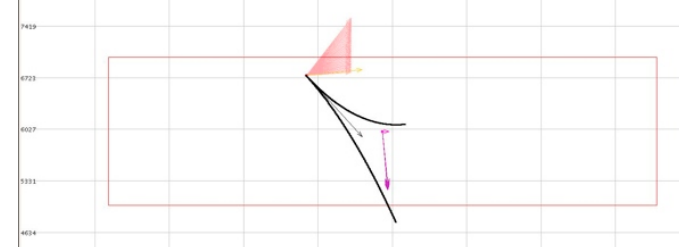

Figure 9. Impact of $1 / 2$ Acceleration On Vertical Maneuvers

\section{Unnecessary Maneuvers}

In Figure 10a we see a situation where a fairly dramatic vertical maneuver is required. In this figure, the rectangle is the protection zone that contains both the ownship (black) and the traffic aircraft (magenta). The criteria region is displayed in pink.

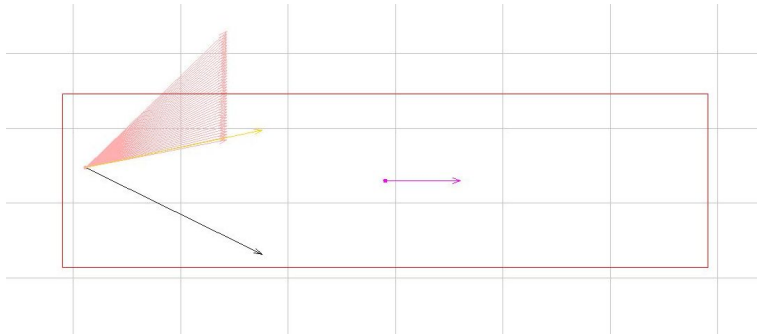

(a) $0 \mathrm{sec}$

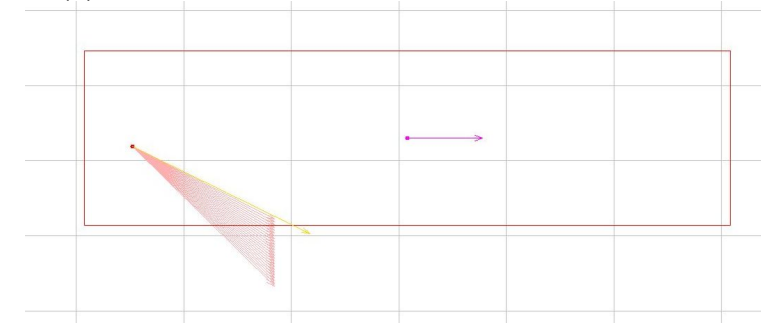

(b) $+10 \mathrm{sec}$

Figure 10. Momentum Switch

The solution space contains only positive vertical speeds even though the current vertical speed is negative. Ironically, the situation will change dramatically in less than 10 seconds, as shown in Figure $10 \mathrm{~b}$. Here the criteria demand a negative vertical speed. The basic issue is that the criteria will produce vastly different guidance due to the momentum of the aircraft. In this situation, the negative solution is clearly better, but our published divergent criteria chooses the positive vertical speed solutions.

\section{Rapid Resolution Change During Transition from Conflict to LoS}

As an aircraft closes in on a protection zone of another aircraft, the maneuvers needed to avoid a loss of separation become increasingly severe. This situation is illustrated in Figure 11. 


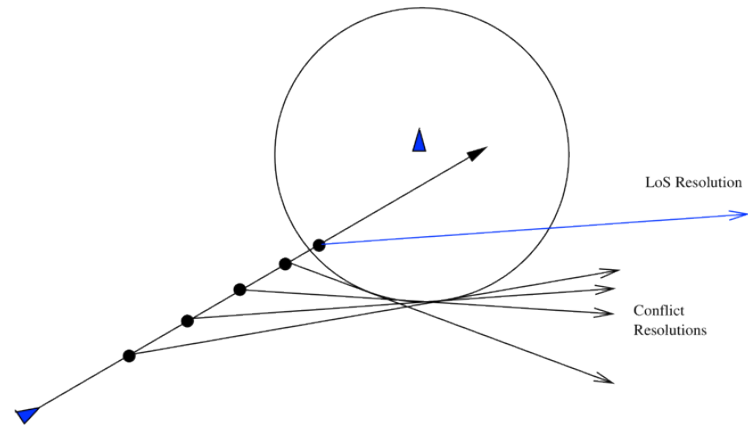

Figure 11. Resolution with Instantaneous Turn Model

Once the aircraft enters the protection zone, a less severe maneuver is immediately obtained because the objective becomes to recover from loss of separation. The pilot is confronted with a large change in the resolution maneuver. Even when the resolutions are in the same direction, this sudden large relaxation in maneuver severity can be confusing to a pilot.

\section{Continuity of Solutions Over Time and The Impact of Critical Points}

If the track angle of one aircraft is changed, the conflict resolutions will also change. In fact in certain critical situations, the resolution can switch from a turn right to a turn left maneuver. This is illustrated in Figure 12 where an aircraft has slight fluctuations in its track angle (possibly due to sensor noise) over a 3 second time period. In each case, the black arrow represents the actual velocity of the aircraft. In

Figure 12a the current ownship track is $229^{\circ}$ and the resolution is a turn left resolution that causes the aircraft to cross in front of the other aircraft. In Figure $12 \mathrm{~b}$, one second later, the current track of the ownship is $230^{\circ}$ and the resolution is a right turn that causes the aircraft to cross behind the other aircraft.

One second later, back at $229^{\circ}$, the suggested resolution again is to turn left, as seen in Figure 12c.

There clearly must be a track angle where the switch occurs; in this case it occurs at track 230 . We call this track a critical point. Critical points occur in both the horizontal and vertical dimensions and in both conflict and loss of separation situations. If an aircraft's current direction is precisely at a critical point, then a minor change in the track can cause a switch from a turn right resolution to a turn left resolution, or vice versa.

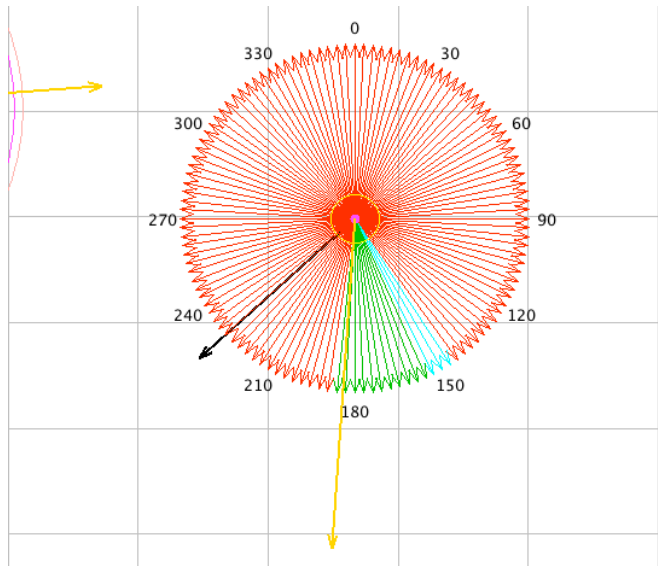

(a) $0 \mathrm{sec}, 229^{\circ}$, turn left to $183^{\circ}$

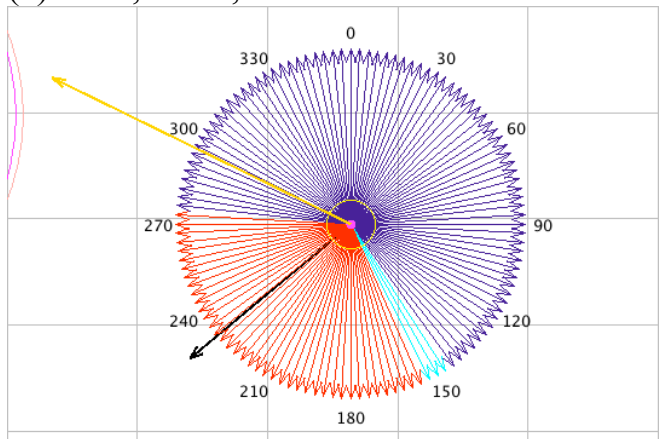

(b) $+1 \mathrm{sec}, 230^{\circ}$, turn right to $291^{\circ}$

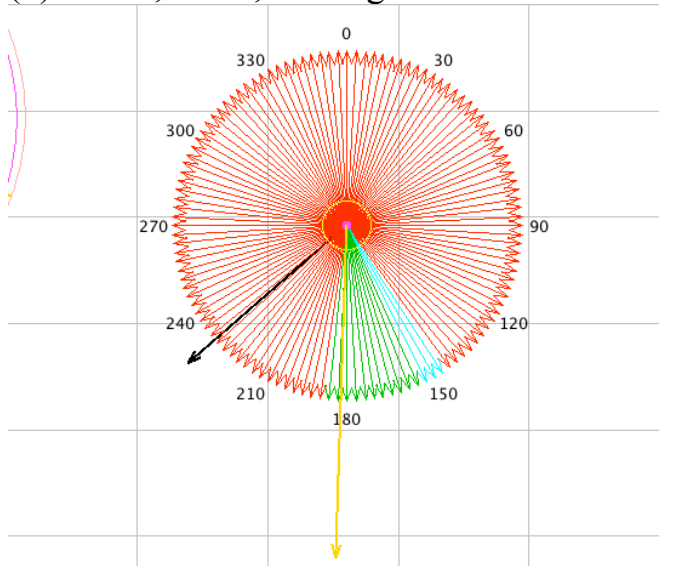

(c) $+2 \sec , 229^{\circ}$, turn left to $183^{\circ}$

\section{Figure 12. Resolutions Near a Critical Point}

If the aircraft stays close to this track but wanders from one side to another, then one can have a rapid oscillation of the turn direction over time. This behavior can be very confusing to a pilot.

\section{A New Approach}

Upon examining the trajectories of the aircraft while maneuvering according to the simple kinematic 
models, it became clear that, in the loss of separation case, the direction of the maneuver is more important than the exact maneuver. In the loss of separation case, the pilot will execute the maximum safe bank angle to achieve the fastest turn possible, the fastest climb, or the fastest descent available. The important thing is that, if both pilots maneuver, then their actions should be coordinated, i.e., the combination of the resolution maneuvers should avoid collision.

For this reason, the previously presented definitions of correctness and the loss of separation criteria are too constraining. In our previous work [2, $3]$, the notion of correctness consisted of two components (1) the maneuver resulted in divergence, and (2) the maneuver resulted in a timely exit from loss of separation. The new criteria presented here are based on the concept of repulsion.

\section{Repulsive Criteria For LoS Recovery}

The concept of repulsion, presented below, is based on the idea that to recover from loss of separation, maneuvers should continually improve the situation while the maneuver is being executed rather than just the final state being an improvement over the current situation. This is due to the relative proximity of aircraft that are in a loss of separation, which makes the intermediate states, between the current time and the time when a resolution is achieved, more important. The mathematical concept of repulsion is presented in [4].

The new horizontal criterion for loss of separation recovery is defined as follows.

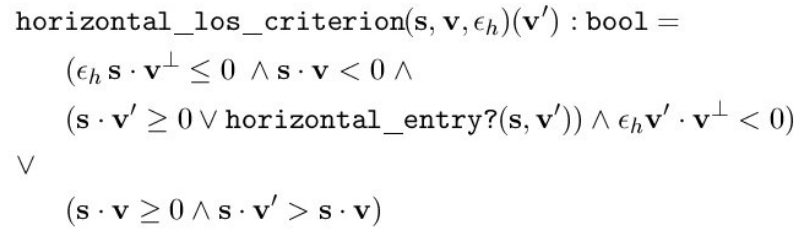

The new vertical criterion for implicitly coordinated loss of separation recovery is defined as follows:

$$
\begin{aligned}
& \text { vertical_los_criterion?(s, v, } \left.\epsilon_{v}, \operatorname{minRelVspd}\right)\left(\mathbf{v}^{\prime}\right)= \\
& \left(\left|s_{z}\right|<H\right) \wedge \\
& \left(\text { IF } \epsilon_{v} v_{z}>0\right. \text { THEN } \\
& \epsilon_{v} v_{z}^{\prime} \geq \epsilon_{v} v_{z} \wedge-\epsilon_{v} v_{z}\left(\mathbf{v}^{\prime} \cdot \mathbf{v}\right)+\epsilon_{v} v_{z}^{\prime}(\mathbf{v} \cdot \mathbf{v}) \geq 0 \\
& \text { ELSE } \\
& \left.\epsilon_{v} v_{z}^{\prime} \geq 0\right) \wedge \\
& \left(\text { IF } \epsilon_{v} v_{z} \leq 0\right. \text { THEN } \\
& \epsilon_{v} v_{z} \geq \operatorname{minRelVspd} \\
& \text { ELSE } \\
& \left.\epsilon_{v} v_{z} \geq \max \left(\operatorname{minRelVspd},\left|v_{z}\right|\right)\right)
\end{aligned}
$$

In this formula, minRelvSpd is a constant parameter representing an arbitrary positive minimum relative vertical speed. Furthermore, the dot products are two-dimensional dot products.

\section{Choice of Direction Parameters, $\varepsilon_{h}$ and $\varepsilon_{v}$}

The criteria presented in the previous section use two direction coordination parameters $\varepsilon_{h}$ and $\varepsilon_{v}$, which take on values \pm 1 . In our figures, green vectors indicate an $\varepsilon_{h}$ of +1 and blue vectors indicate an $\varepsilon_{h}$ of -1 . These parameters capture the notions of turning direction in the horizontal dimension and upor-down in the vertical dimension. ${ }^{2}$ From the standpoint of the criteria, in most cases, the choice is arbitrary, and either choice is safe. Therefore, we can choose the epsilon values based on other factors, such as minimizing the size of the turn.

Although there are many schemes that could be developed for the horizontal parameter $\varepsilon_{h}$, this parameter must satisfy the following property:

$$
\varepsilon_{h}(\mathbf{s}, \mathbf{v})=\varepsilon_{h}(-\mathbf{s},-\mathbf{v}) .
$$

We propose the following formula:

$$
\varepsilon_{h}=\operatorname{sign}\left(\mathbf{s} \cdot \mathbf{v}^{\perp}\right) .
$$

The parameter value $\varepsilon_{v}$ for the vertical criterion must satisfy the following property:

$$
\varepsilon_{v}(\mathbf{s}, \mathbf{v})=-\varepsilon_{v}(-\mathbf{s},-\mathbf{v})
$$

\footnotetext{
${ }^{2}$ Note that these parameters determine direction in a relative frame. The actual maneuver when $\varepsilon_{h}=+1$, for example, could be either a left or a right turn from the pilot's point of view, depending on the situation.
} 
The vertical loss direction parameter is defined using a smaller inner collision zone. We propose the following formula:

$$
\left.\epsilon_{v}(\mathbf{s}, \mathbf{v}) \equiv \text { break_symm(vertical_decision_vect }(\mathbf{s}, \mathbf{v})\right)
$$

where vertical_decision_vect(s, $\mathbf{v})$ is defined by cases:

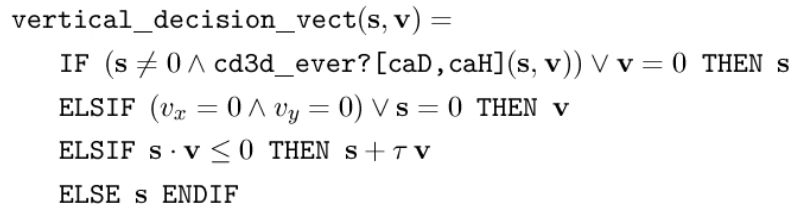

In this formula, the dot product is two-dimensional and $\tau=\frac{-\mathbf{s} \cdot \mathbf{v}}{v^{2}}$. The predicate cd3d_ever? is a threedimensional conflict probe that tests if the aircraft are in conflict with an inner collision zone of diameter $\mathrm{caD}$ and height $\mathrm{caH}$. The multiple branches cover different cases. The third branch is the key branch that covers most cases. It is continuous over time if the aircraft velocity vectors are constant. The break_symm function is required to handle the perfectly symmetrical case where the altitudes are equal and the vertical speed is 0 . This function picks opposite directions for the ownship and traffic.

\section{The New Repulsive Algorithms}

The new repulsive algorithms use iteration to find solutions. They search in the direction that is repulsive and stop the search when a divergent velocity vector or a non-repulsive vector is reached. The following functions define the notion of repulsiveness for track maneuvers:

$$
\begin{aligned}
& \text { right_turn_repulsive? }\left(\mathbf{s}, \mathbf{v}_{\mathbf{o}}, \mathbf{v}_{\mathbf{i}}\right)= \\
& \text { repulsive_along? } \left.\left(\mathbf{s}, \mathbf{v}_{\mathbf{o}}-\mathbf{v}_{\mathbf{i}}\right)\left(\mathbf{v}_{\mathbf{o}}{ }^{\perp}\right)\right) \\
& \text { left_turn_repulsive? }\left(\mathbf{s}, \mathbf{v}_{\mathbf{o}}, \mathbf{v}_{\mathbf{i}}\right)= \\
& \text { repulsive_along? } \left.\left(\mathbf{s}, \mathbf{v}_{\mathbf{o}}-\mathbf{v}_{\mathbf{i}}\right)\left(-\mathbf{v}_{\mathbf{o}}{ }^{\perp}\right)\right)
\end{aligned}
$$

where repulsive_along? is defined as

$$
\begin{aligned}
& \text { repulsive_along?(s,v) }\left(\mathbf{v}^{\prime}\right)= \\
& \left(\mathbf{s} \cdot \mathbf{v}<0 \wedge-\operatorname{sign}\left(\mathbf{s} \cdot \mathbf{v}^{\perp}\right)\left(\mathbf{v}^{\prime} \cdot \mathbf{v}^{\perp}\right)<0\right) \\
& \vee\left(\mathbf{s} \cdot \mathbf{v} \geq 0 \wedge \mathbf{s} \cdot \mathbf{v}^{\prime}>0\right)
\end{aligned}
$$

The repulsive track algorithm chooses a starting track and a search direction based on the current criteria, and then iteratively checks successively larger resolution turns in that direction. It continues until it reaches a maneuver that is either divergent or no longer repulsive, and then stops, returning that maneuver. For the kinematic version, the algorithm advances time as part of this iterative search, at each step linearly projecting the traffic and recalculating the position and velocity of the ownship along the turn before determining the maneuver's divergence or repulsiveness. The search terminates once a $180^{\circ}$ turn is reached, if it has not found an earlier solution.

After a solution is found for the most urgent conflict, the search will continue over the remaining unexplored repulsive tracks until all secondary conflicts are avoided as well. If this is not possible then the solution for the most urgent aircraft is returned. A flag is set if the kinematic search enters a LoS with another aircraft before it is able to find a resolution for the primary.

The ground speed and vertical speed algorithms are nearly identical, with user-defined velocity limits for the ownship. A forthcoming NASA report will fully document these algorithms.

\section{Achieving Continuity}

Under the stress of a near term loss of separation, pilots may become confused if the resolutions change too fast. Therefore, it is important that CD\&R algorithms do not switch from turn right to turn left (and vice versa) or from go up to go down (or vice versa) solutions too quickly as time progresses. We call this property continuity.

\section{Continuity Into LoS}

At some point during the entry into LoS, the track maneuver becomes infeasible, i.e. the aircraft is not able to complete the proposed maneuver without entering LoS. At this point it is probably more prudent to produce a resolution that is consistent with the resolution that will be issued once LoS has occurred. The goal is no longer to avoid loss of separation, but rather to minimize the depth of the entry.

In these cases we instead project the state vectors linearly to a time exactly $1 / 2$ second after entry into LoS. We then use the LoS resolution algorithms on this time-projected data. 


\section{Continuity Near A Critical Point}

When an aircraft's current state is located near a critical point, rapidly changing resolutions are possible as time progresses. This is due to slight variations in the track due to external disturbances and the inability of any system to perfectly follow a track. There are several possible approaches to dealing with this problem:

- Use dead bands. In this case, the algorithm freezes the resolutions while the aircraft stays on a critical point.

- Filter the direction. In this case, the algorithm freezes the direction of the resolutions.

- Use future resolutions. In this case, the algorithm computes a resolution in the future and holds it as long as it is still valid.

We have currently settled on a variation of the second option, and are using a hysteresis filter that dampens the fluctuation between directions. Rapid changes over a short time period are ignored, preserving earlier calculated direction and coordination parameters. In the event an actual change of direction is needed once the critical point has been left, this registers after a few seconds.

\section{Revisiting Earlier Examples Using the New Algorithms}

The maneuvers of Figure 2 were excessive due to the divergence criteria. The new criteria produce the results shown in Figure 13. The gold vectors represent divergent vectors.

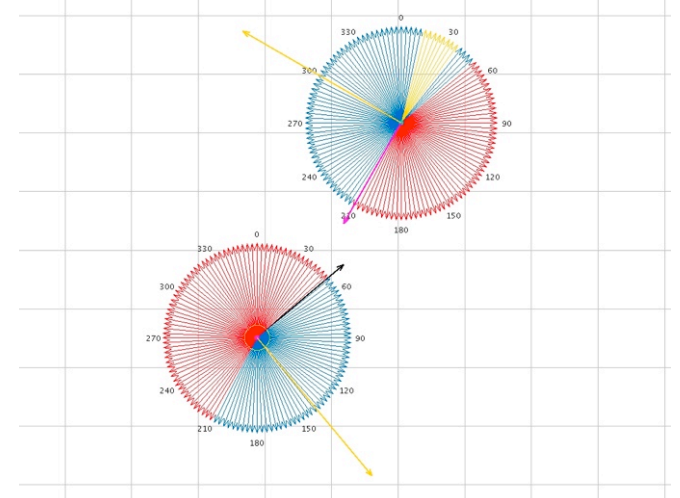

Figure 13. Extreme Maneuvers Problem Solved
In Figure 4, we saw a situation where no horizontal maneuvers were obtained. The new criteria produce the results shown in Figure 14.

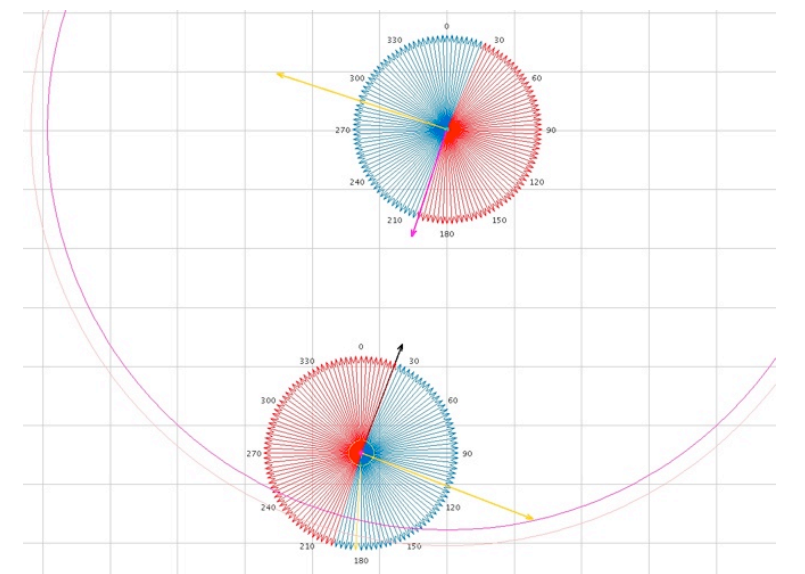

Figure 14. No Horizontal Solution Problem Solved

In Figure 6, we saw an example where original criteria solutions brought the aircraft within $.01 \mathrm{~nm}$ of each other. The repulsive criteria algorithms produced the results shown in Figure 15.

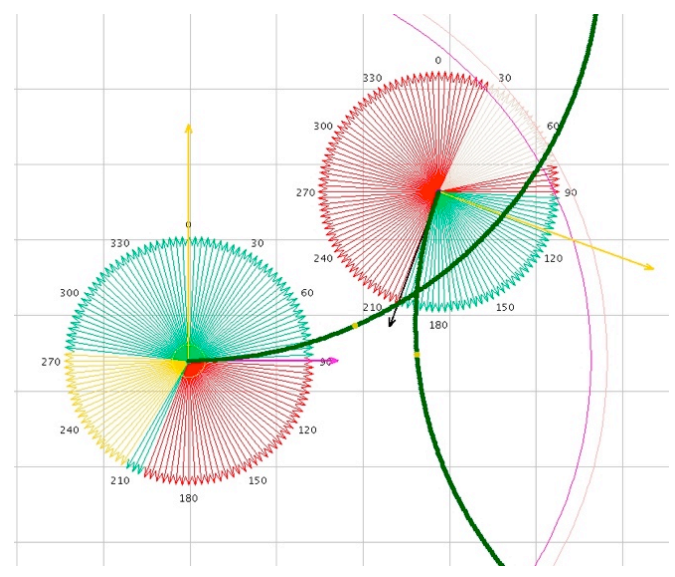

Figure 15. Close Encounter Problem Solved

The distance at closest approach is $0.84 \mathrm{~nm}$. The gold dot on the future trajectories shows the point of closest approach.

In Figure 10a, we saw a situation where the original criteria resulted in a fairly dramatic vertical maneuver. In Figure 16, we see the repulsive criteria in action. Here the aircraft is allowed to continue its descent. 


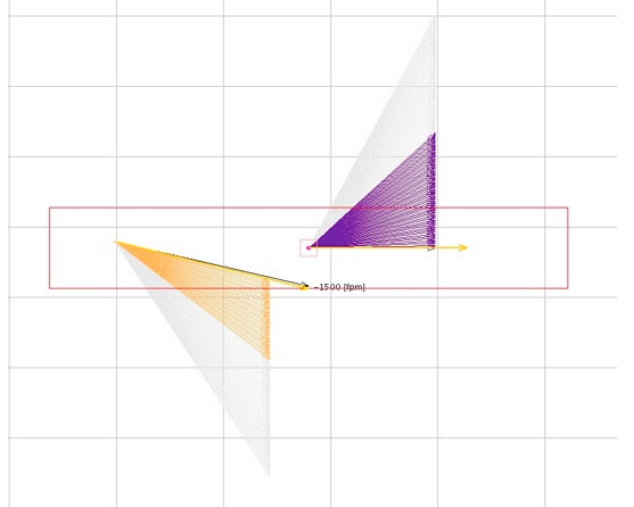

Figure 16. Momentum Switch Problem Solved

In Figure 11, we saw a case where track resolutions became more and more severe until the ownship entered LoS, and then immediately relaxed. In Figure 17, we see a possible result of detecting the infeasibility of conflict avoidance resolutions and adopting a LoS resolution before actually entering loss of separation.

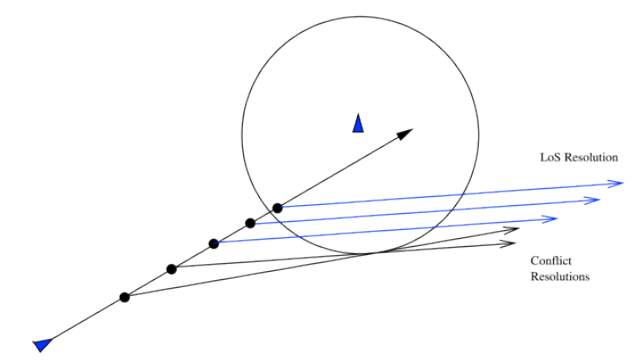

Figure 17. Resolution with Turn Model

In Figure 18, we see that even with track fluctuations the resolutions remain constant.

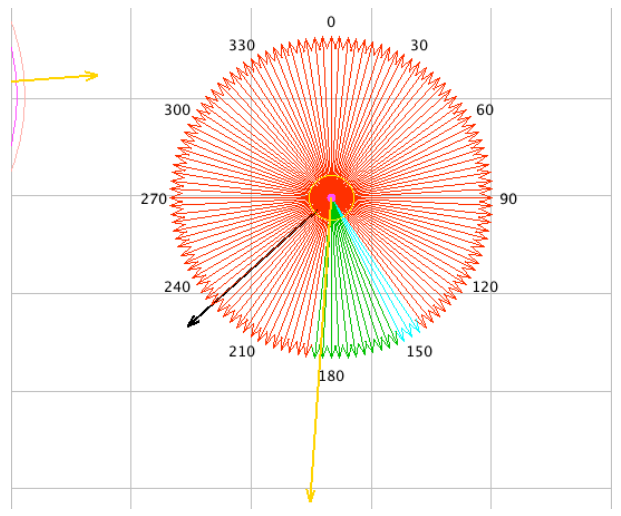

(a) $0 \mathrm{sec}, 229^{\circ}$, turn left to $183^{\circ}$

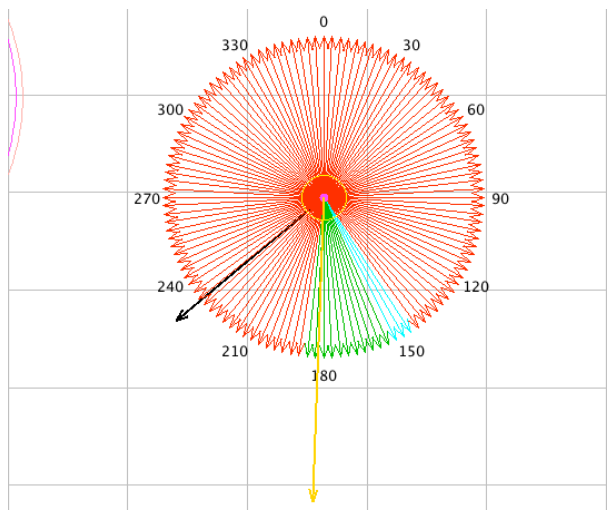

(b) $+1 \mathrm{sec}, 230^{\circ}$, turn left to $183^{\circ}$

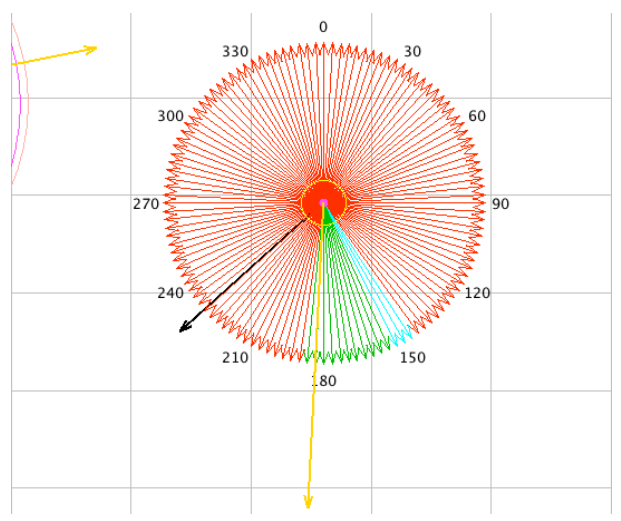

(c) $+2 \mathrm{sec}, 229^{\circ}$, turn left to $183^{\circ}$

Figure 18. Resolutions Near a Critical Point 


\section{Conclusion}

This paper revisits previous work $[2,3,5]$ on the development of criteria for implicitly coordinated loss of separation recovery maneuvers. The criteria previously proposed are based on the concepts of divergence and timeliness of the recovery maneuvers. Extensive experimentation has shown that even though that approach was satisfactory in many cases, it relied on overly optimistic assumptions, and thus resulted in poor performance in other cases.

In this paper, new horizontal and vertical loss of separation recovery criteria are proposed. The new criteria are based on the concept of repulsiveness. Experimentation has shown that the new criteria successfully solves most of the problems encountered with the original approach.

\section{References}

[1] Wing, David and William Cotton, 2011, Autonomous flight rules a concept for self-separation in U.S. domestic airspace. Technical Publication NASA/TP-2011-217174, NASA, Langley Research Center, Hampton VA 23681-2199, USA.

[2] Butler, Ricky, César Muñoz, 2008, A formal framework for the analysis of algorithms that recover from loss of separation, Technical Memorandum NASA/TM-2008-215356, NASA, Langley Research Center, Hampton VA 23681-2199, USA.

[3] Butler, Ricky, César Muñoz, 2009, Formally verified practical algorithms for recovery from loss of separation. Technical Memorandum NASA/TM2009-215726, NASA, Langley Research Center, Hampton VA 23681-2199, USA.

[4] Narkawicz, Anthony, César Muñoz, 2011, Statebased implicit coordination and applications. Technical Publication NASA/TP-2011-217067, NASA, Langley Research Center, Hampton VA 23681-2199, USA.

[5] Muñoz, César, Ricky Butler, Anthony Narkawicz, Jeffrey Maddalon, and George Hagen, A Criteria Standard for Conflict Resolution: A Vision for Guaranteeing the Safety of Self-Separation in NextGen, Technical Memorandum, NASA/TM2010-216862, October 2010.

31st Digital Avionics Systems Conference

October 14-18, 2012 